\title{
TIPIFICACIÓN SARM ENTRABAJADORES ASISTENCIALES DE LAS UNIDADES DE CUIDADOS INTENSIVOS EN UNA INSTITUCIÓN HOSPITALARIA
}

\section{MRSA TYPING IN HEALTH CARE WORKERS FROM INTENSIVE CARE UNITS OF A HOSPITAL INSTITUTION}

\author{
Maria Eugenia Ferreira $\mathrm{C}^{1}$, Karina Valencia $\mathrm{J}^{2}$, Francisco Alberto Buelvas $\mathrm{D}^{3}$, Catalina Tovar $\mathrm{A}^{4}$, Mayra Ligia \\ Raciny $A^{5 *}$.
}

Recibido para publicación: Febrero 14 de 2017 - Aceptado para publicación: Mayo 3 de 2017

\begin{abstract}
RESUMEN
Objetivo: Caracterizar microbiológicamente SARM en trabajadores asistenciales de las Unidades de Cuidados Intensivos en una institución hospitalaria de la ciudad de Montería. Materiales y métodos: Se realizó un estudio descriptivo longitudinal, en 52 trabajadores de dos unidades de cuidado intensivo; se tomaron hisopados nasales y faríngeos a cada individuo, con una periodicidad de 15 días/cinco meses. Mediante pruebas convencionales como fermentación de manitol, catalasa y coagulasa se realizó la identificación de $S$. aureus, con el disco de cefoxitín por KirbyBauer se evaluó la resistencia a Meticilina mediada por el gen mecA; antibióticos como Vancomicina, Eritromicina y Clindamicina fueron evaluados por esta prueba y confirmados por MicroScan ${ }^{\circledR}$. Resultados: se obtuvieron 88 aislamientos de $S$. aureus y 21 aislamientos SARM, una prevalencia de portación de SARM del $25 \%$. El $90,5 \%$ de las cepas de SARM fueron recuperadas de la faringe, en mayor proporción (50\%) en el personal de enfermería. Conclusiones: La evidencia de su circulación de SARM en el personal asistencial dentro de una institución hospital, genera una alerta que le permite a la misma establecer políticas de contención para evitar la diseminación de estas cepas y evitar la aparición de broteso infecciones hospitalarias asociadas a este patógeno.
\end{abstract}

Palabras clave: Staphylococcus aureus, SARM, Cefoxitín, Infecciones intrahospitalarias, Portador.

\begin{abstract}
Objective: To characterize microbiologically MRSA in health care workers of the Intensive Care Units at a hospital institution in the city of Montería. Materials and methods: A longitudinal descriptive study was carried out in 52 workers from two intensive care units; nasal and pharyngeal swabs were taken from each individual, with a periodicity of 15 days / five months. Examined through microbiological methods such as fermentation of mannitol, catalase and coagulase the identification of $S$. aureus was performed, with the cefoxitin disc by Kirby-Bauer disc-diffusion method the methicillin resistance mediated by the mecA gene was evaluated; antibiotics such as Vancomycin, Erythromycin and Clindamycin were evaluated by this test and confirmed by MicroScan ${ }^{\oplus}$. Results: we obtained 88 isolates of Staphylococcus aureus and 21 MRSA, a prevalence of MRSA of $25 \% .90 .5 \%$ of strains Of SARM were recovered from the pharynx, in a greater proportion $(50 \%)$ in nursing staff. Conclusions: Evidence of MRSA circulation in care staff within a hospital institution generates an alert that allows it to establish containment policies to avoid the dissemination of these strains and to avoid the appearance of outbreaks or hospital infections associated with this pathogen.
\end{abstract}

Key words: Staphylococcus aureus, MRSA, cefoxitin, Cross Infection, Carrier State. infections associated with health care (IACS), nasal carriers.

\footnotetext{
${ }^{1}$ Bacterióloga. Universidad de Córdoba

${ }^{2}$ M.Sc.Grupo de investigación en Enfermedades Tropicales y Resistencia Bacteriana. Universidad del Sinú ${ }^{3}$ M.Sc.Grupo de investigación en Enfermedades Tropicales y Resistencia Bacteriana. Universidad del Sinú ${ }^{4}$ M.Sc.Grupo de investigación en Enfermedades Tropicales y Resistencia Bacteriana. Universidad del Sinú ${ }^{5}$ M.Sc. Docente Programa de Bacteriología, Universidad de Córdoba. Grupo de Investigaciones Microbiológicas y Biomédicas de Córdoba - GIMBIC - mracinyaleman@correo.unicordoba.edu.co
} 


\section{INTRODUCCIÓN}

Staphylococcus aureus es un patógeno que causa infecciones nosocomiales y comunitarias, puede colonizar las fosas nasales anteriores, faringe y otros sitios anatómicos en menor proporción (1) Staphylococcus aureus resistente a la meticilina (SARM) en la actualidad es uno de los principales problemas de salud, por presentar elevadas tasas de morbimortalidad y cepas que exhiben perfiles de resistencia a diferentes grupos de antimicrobianos. Los pacientes hospitalizados y el personal asistencial constituyen un reservorio de cepas de SARM, que se pueden convertir en una fuente importante de infección para pacientes susceptibles y de vehículo de transmisión dentro y fuera de la institución de microorganismos con estos perfiles de resistencia (2).

La adquisición de esta resistencia en $S$. aureus se debe principalmente a la presencia del gen $m e c A$, el cual codifica para una proteína de unión a la penicilina (PBP), llamada PBP2A, que presenta baja afinidad por la meticilina y todos los antibióticos $\beta$ - lactámicos que se han desarrollado, adicionalmente se presenta resistencia cruzada con antibióticos como Eritromicina, Estreptomicina, Tetraciclina, Vancomicina, entre otros; como consecuencia de la integración de plásmidos y transposones en el casete cromosomal SCC mec (3).

Diferentes estudios han mostrado que la colonización con SARM precede a la infección, casos específicos se han reportado donde pacientes que previamente colonizados desarrollan infecciones de piel y tejidos blandos a mediano plazo, incluso se han reportado infecciones como celulitis, impétigo, foliculitis y conjuntivitis en personal asistencial anticipadamente colonizado con SARM (4). Así mismo se han desarrollado estudios evaluando la descolonización de los portadores con antimicrobianos, y la adherencia a algunas medidas antisépticas vs la persistencia de la colonización por SARM y la aparición de infecciones por este microorganismo, en algunos casos se muestra una alta tasa de efectividad post intervención y en otros estudios reportan la presencia de portadores persistentes (5). Se recomienda así la vigilancia activa de SARM con miras a un seguimiento intensivo que permita implementar políticas que frenen la diseminación y la reducción de infecciones nosocomiales (6). Con el fin de conocer la epidemiología local se planteó caracterizar microbiológicamente SARM en trabajadores asistenciales de dos Unidades de Cuidados Intensivos en una institución hospitalaria de la ciudad de Montería.

\section{MATERIALES Y METODOS}

Se realizó un estudio descriptivo longitudinal durante el cual se incluyeron 52 trabajadores asistenciales de la Unidad de Cuidado Intensivo adulto y/o neonatal de una institución hospitalaria. Se recolectaron hisopados nasales y faríngeos de cada individuo con una periodicidad de 15 días hasta completar 10 muestreos por individuo. Las muestras fueron analizadas por métodos convencionales de microbiología como fermentación del manitol, coloración de Gram, determinación de la presencia de catalasa y coagulasa, para la identificación de $S$. aureus. Los aislamientos identificados y confirmados como $S$. aureus fueron analizados por test de difusión de disco de cefoxitín de $30 \mu \mathrm{g}$ para predecir la resistencia mediada por el gen mecA: la prueba se interpretó como positiva con un halo $\leq 21 \mathrm{~mm}$ para $S$. aureus (CLSI 2008, Volumen lizar p27, Table2C, Staphylococcus spp. M2-Disk Difusión). Adicionalmente por la técnica de KirbyBauer se analizó el perfil de susceptibilidad frente Oxacilina $(1 \mu \mathrm{g})$, Clindamicina $(2 \mu \mathrm{g})$, Eritroicina $(15 \mu \mathrm{g})$, Vancomicina $(30 \mu \mathrm{g})$ y Mupirocina $(200$ $\mu g)$. Se realizó la confirmación de especie y la susceptibilidad por Concentración Mínima Inhibitoria (CIM) empleando el sistema automatizado MicroScam $\mathrm{PC} \mathrm{A}^{\circledR}$ 
(DadeBehring, CA, USA). Como cepas control se utilizaron $S$. aureus ATCC ${ }^{\circledR}$ 25923, S. aureus ATCC $^{\circledR}$ 43300, según las recomendaciones del CLSI 2008 (7). Los datos epidemiológicos fueron tabulados en Excel y los datos microbiológicos se analizaron e interpretaron en el software Labpro. Se elaboraron tablas dinámicas en Excel que permitieron realizar el conteo de cada una de las variables y se determinaron frecuencias, porcentajes y promedios. Se analizó la información y se elaboraron tablas y gráficas para una mejor presentación y comprensión de los resultados

Para la clasificación por tipo de portación se tuvieron en cuenta los criterios propuestos por algunos autores, $(8,9,10)$ con algunas adaptaciones (Tabla 1):

Tabla 1. Criterios de clasificación para la portación de SARM

\begin{tabular}{|c|c|c|c|}
\hline Tipo de portación & Definición & Criterios de clasificación & Tipo de criterio \\
\hline No portador & $\begin{array}{l}\text { Persona que no presentó } S \text {. aureus o } \\
\text { SARM en algún sitio anatómico (nasal } \\
\text { faringe). }\end{array}$ & $\begin{array}{l}\text { Todos los aislamientos } \\
\text { negativos para } S \text {. aureus o } \\
\text { SARM. }\end{array}$ & Critierio 1 \\
\hline Portador intermitente & $\begin{array}{l}\text { Persona que presentó } S \text {. aureus o SARM } \\
\text { en algún sitio anatómico pero que no en } \\
\text { la totalidad de los muestreos. }\end{array}$ & $\begin{array}{l}\text { 1-8 aislamiento positivos } \\
\text { para } S \text {. aureus o SARM. }\end{array}$ & Critierio 2 \\
\hline Portador persistente & $\begin{array}{l}\text { Persona que en todo momento presentó } S \text {. } \\
\text { aureus o SARM en algún sitio anatómico. }\end{array}$ & $\begin{array}{l}\text { 9-10 aislamiento positivos } \\
\text { para } S \text {. aureus o SARM. }\end{array}$ & Critierio 3 \\
\hline
\end{tabular}

De acuerdo con la Resolución $\mathrm{N}^{\circ} 008430$ de 1993 , en la que se establecen las normas científicas, técnicas y administrativas para la investigación en salud en Colombia, este estudio se clasificó con riesgo mínimo por incluir solo procedimientos con baja probabilidad de afectar a los individuos participantes, tales como la toma y recolección de hisopados nasales y faríngeos. La ejecución de los mismos fue realizada por profesionales idóneos preservando la integridad de los individuos participantes. Se obtuvo el consentimiento informado por escrito de cada uno de ellos, así como la autorización de la entidad de salud involucrada. Se mantuvo la privacidad y el anonimato de los individuos participantes y la despersonalización de los datos.

\section{RESULTADOS}

De los 52 trabajadores vinculados en el estudio, 33 pertenecían a la $\mathrm{UCl}$ de adultos y 19 a la $\mathrm{UCl}$ de neonatos. En total se procesaron 966 muestras, provenientes de los 52 individuos participantes, la prevalencia de $S$. aureus fue de $84,6 \%(44 / 52)$ y para $S$. aureus resistente a meticilina (SARM) de $25 \%(13 / 52)$, se obtuvo un total de 21 cepas de SARM, algunas de ellas aisladas de un mismo individuo en periodos de tiempo diferente. El $90.5 \%$ de las cepas de SARM (19/21) fueron aisladas a partir de las muestras nasales, siendo el personal de enfermería el mayor porcentaje de portación (Tabla 2). 
AVANCES EN SALUD - Vol. I:(I) Enero - Junio 2017 (7 - I3)

Tabla 1. Porcentajes de portación de $S$. aureus y de SARM en personal asistencial

\begin{tabular}{|c|c|c|c|c|c|c|}
\hline \multirow{2}{*}{ Cargo } & \multicolumn{2}{|c|}{ No personas por UCI } & \multicolumn{2}{|c|}{ Porcentaje S. aureus (\%) } & \multicolumn{2}{|c|}{ Porcentaje de SARM (\%) } \\
\hline & Adulto & Neonatal & Adulto & Neonatal & Adulto & Neonatal \\
\hline Enfermeras & 7 & 2 & 100 & 50 & 42,9 & 50 \\
\hline Auxiliares de enfermería & 19 & 12 & 68 & 100 & 31,6 & 16,7 \\
\hline Médico Intensivista/ Pediatra & 3 & 4 & 100 & 75 & 0 & 25 \\
\hline Fisioterapeuta & 3 & 1 & 100 & 100 & 0 & 0 \\
\hline Nutricionista & 1 & & 100 & & 0 & 0 \\
\hline
\end{tabular}

En el análisis de susceptibilidad a los antimicrobianos por difusión de disco de las cepas de SARM se encontró que $31 \%$ de las cepas presentaron un perfil de resistencia a Eritromicina del $63 \%$ y del $43 \%$ frente a Clindamicina. Por CIM se encontró un perfil de resistencia del $100 \%$ frente a los antibióticos del grupo de $\beta$-lactámicos, el $66.7 \%$ de resistencia frente al grupo de los Macrólidos-LincosamidasEstreptograminas (MLS) y en menor proporción para otros antibióticos como la Gentamicina y el Trimetoprim/Sulfametoxazol. Para los demás antibióticos evaluados se halló un sensibilidad del $100 \%$ (Figura 1).
De acuerdo a los criterios de evaluación del tipo de portación de $\mathrm{S}$. aureus se encontró que el $76,9 \%(40 / 52)$ de los trabajadores fueron portadores intermitentes y el $23,1 \%$ (12/52) fueron no portadores, sin hallazgos de portadores persistentes. En cuanto a SARM se encontró que el $25 \%(13 / 52)$ fueron portadores intermitentes y el $75 \%(39 / 52)$ fueron no portadores, sin hallazgos de portadores persistentes (Tabla 3).

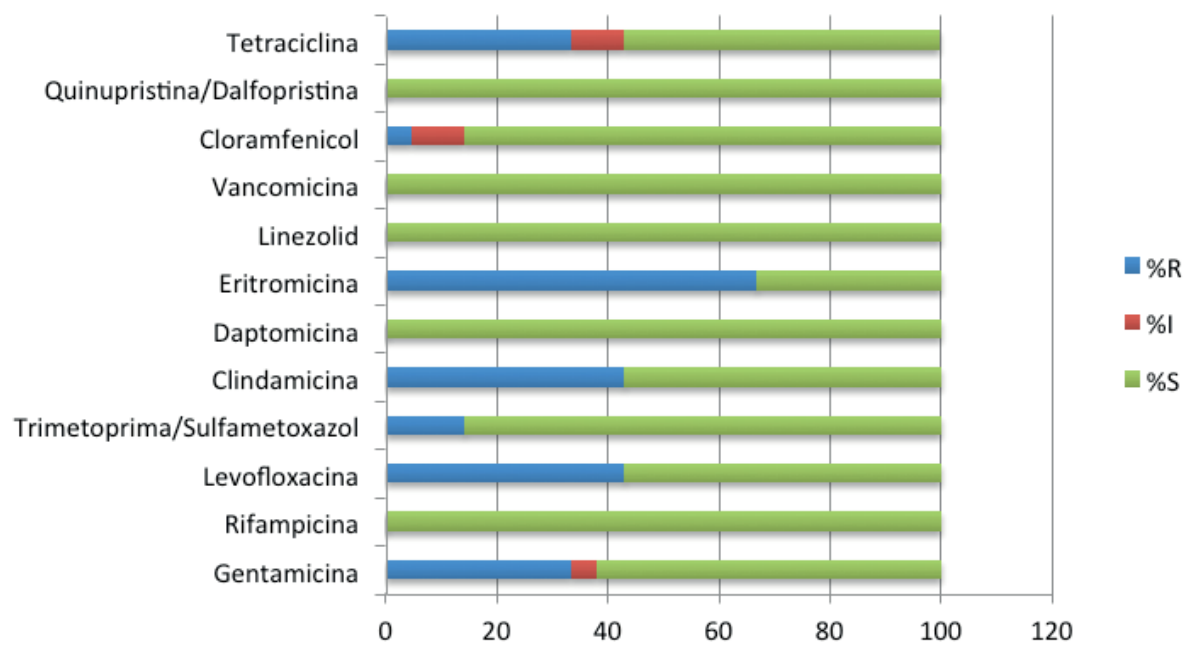

Figura1. Perfiles de susceptibilidad de los aislamientos de SARM

R: resistente, I: intermedio, S: sensible 
Ferreira et. al. - Tipificación sarm en trabajadores asistenciales de las unidades de cuidados intensivos

Tabla 3. Discriminación del tipo de portación en el personal asistencial

\begin{tabular}{|c|c|c|c|c|c|c|c|c|c|c|c|c|c|}
\hline \multirow{3}{*}{ Tipo de portación } & \multirow{3}{*}{ Criterio } & \multicolumn{4}{|c|}{ S. aureus } & \multicolumn{4}{|c|}{ SARM } & \multicolumn{4}{|c|}{ TOTAL } \\
\hline & & \multicolumn{2}{|c|}{ UCI } & \multicolumn{2}{|c|}{ CIREN } & \multicolumn{2}{|c|}{ UCI } & \multicolumn{2}{|c|}{ CIREN } & \multicolumn{2}{|c|}{ UCI } & \multicolumn{2}{|c|}{ CIREN } \\
\hline & & $\mathbf{N}$ & $\%$ & $\mathbf{N}$ & $\%$ & $\mathbf{N}$ & $\%$ & $\mathbf{N}$ & $\%$ & $\mathbf{N}$ & $\%$ & $\mathbf{N}$ & $\%$ \\
\hline No portador & 1 & 10 & 30,3 & 2 & 10,5 & 24 & 72,7 & 15 & 78,9 & 12 & 23,1 & 39 & 75 \\
\hline Intermitente & 2 & 23 & 69,7 & 17 & 89,5 & 9 & 27,3 & 4 & 21,1 & 40 & 76,9 & 13 & 25 \\
\hline Persistente & 3 & 0 & 0,0 & 0 & 0,0 & 0 & 0,0 & 0 & 0,0 & 0 & 0,0 & 0 & 0 \\
\hline TOTAL & & 33 & & 19 & & 33 & & 19 & & & & & \\
\hline
\end{tabular}

Criterio 1: ningún aislamiento positivo para S. aureus o SARM; Criterio 2: 1-8 aislamientos positivos para $S$. aureus SARM; Criterio 3: 9-10 aislamiento positivos para $S$. aureus o SARM.

\section{DISCUSIÓN}

Las cepas de SARM constituyen un grave problema clínico y epidemiológico, por el incremento de infecciones y cepas que son a menudo multirresistentes (11). Las tasas de portación tanto de $S$. aureus como de SARM varían a nivel mundial. De $S$. aureus se conocen tasas de portación hasta del 56\% en personal hospitalario (12), en cuanto a SARM se conocen reportes hasta del $30 \%$. En países latino americanos los datos publicados están alrededor del $17 \%(13)$. En Colombiase conocen reportes que oscilan entre $7 \%$ al $11 \%$, para la comunidad medico asistencial como para la comunidad en general $(14,15)$. En el presente estudio se encontró un nivel de portación del $25 \%$ de SARM en personal asistencial de dos Unidades de Cuidado Intensivo, un porcentaje alto con respecto a otras publicaciones en el tema. Estudios de portación realizados por diferentes autores entre ellos Oliveira (16), refieren una alta tasa de portación intermedia, e identificación de portadores persistentes, en nuestra investigación a pesar de los 10 muestreos que se realizaron en el periodo de evaluación no fueron identificados portadores persistentes, al contrario de haber identificación una tasa de portación considerable en el rango de intermedio. Anatómicamente existió una mayor tasa de portación a nivel de las fosas nasales, sitio reconocido como fuente importante en la diseminación de la infección estafilococcica desde hace décadas, siendo el mejor indicador de la colonización y potencial diseminación del agente. Como dato destacado se considera el aislamiento de tres cepas de SARM en un médico pediatra de la $\mathrm{UCl}$ neonatal, y dos cepas de SARM en un auxiliar de enfermería de la $\mathrm{UCl}$ de adultos, todas estas obtenidas de fosas nasales en periodos de tiempo diferentes, cepas que a futuro serán evaluadas por técnicas moleculares para identificar si son clones idénticos o diferentes, información que ilustraría en parte el comportamiento en cuanto a diseminación de este patógeno en la institución hospitalaria.

En cuanto a los hallazgos de perfiles de susceptibilidad se encontró una respuesta concordante del perfil de resistencia a los

$\beta$ - lactámicos y la presencia del mecanismos de resistencia mediados por el gen mecA. Es de resaltar un porcentaje del $66.7 \%$ de los aislamientos SARM resistentes a antibióticos como clindamicina y eritromicina, resultado diferente a los expuesto por otros autores como Cárdenas O. y Cruz H (17) donde reportan un porcentaje de resistencia frente a este grupo de antibióticos del $25 \%$.

La evaluación fenotípica de la resistencia al resto de antimicrobianos por CIM reporta porcentajes altos de sensibilidad, lo que permite inferir a través del comportamiento fenotípico la presencia presuntiva de cepas de SARM de 
origen comunitario(SARM-AC) resultados que coinciden con el estudio de Cortes Jorge et al (18) en el 2009 en donde realizaron pruebas de susceptibilidad a cepas SARM-AC y SARM$\mathrm{AH}$, identificadas previamente por métodos moleculares, encontrando una sensibilidad de las cepas SARM-AC con un comportamiento similar $(19,20)$. Nosotros informamos esta tendencia a pesar de no existir una concertación con respecto a la definición de estos términos.

\section{CONCLUSIONES}

La portación de SARM en personal asistencial en nivel local reportada en este estudio en uno de los datos más altos descritos en Latinoamérica. El aislamiento de diferentes cepas de SARM de un mismo individuo en periodos de tiempo diferente, requiere estudios moleculares que permitan evaluar la clonalidad, con el fin de saber, si estos individuos son portadores persistentes de un mismo clon o si son portadores de diferentes clones de SARM.

\section{RECOMENDACIONES}

Se recomienda complementar este estudio con análisis moleculares que permitan indagar más acerca de la epidemiología molecular y describir el tipo de clonalidad de estas cepas.

\section{REFERENCIAS BIBLIOGRÁFICAS}

1. Girou E, Pujade G, Legrand P, Cizeau F, Brun-Buisson C. Selective screening of carriers for control of methicillin-resistant Staphylococcus aureus (MRSA) in high-risk hospital areas with a high level of endemic MRSA. Clin Infect Dis 1998; 27(3):543-50.

2. Larson EL, Hughes CA, Pyrek JD, Sparks SM, Cagatay EU, Bartkus JM. Changes in bacterial flora associated with skin damage on hands of health care personnel. Am J Infect Control 1998; 26(5):513-21.
3. Ito T., Katayama $\mathrm{Y}$, Asada K, Mori N, Tsutsumimoto $\mathrm{K}$, Tiensasitorn $\mathrm{C}$, and. Hiramatsu K. Structural comparison of three types of staphylococcal cassette chromosome mec integrated in the chromosome in methicillin-resistant Staphylococcus aureus. Antimicrob Agents Chemother 2001;45 (5): 1323-1336.

4. Mitsuda $T$, Arai $K$, Ibe $M$, Imagawa $T$, Tomono N, Yokota S. The influence of methicillin-resistant Staphylococcus aureus (MRSA) carriers in a nursery and transmission of MRSA to their households. J Hosp Infect 1999; 42(1):45-51.

5. Wagenvoort JH, Toenbrecker HM, Nurmohamed A, Davies BI. Transmission of methicillin-resistant Staphylococcus aureus within a household. Eur J Clin Microbiol Infect Dis 1997; 16:399-400.

6. Safdar N, Marx J, Meyer NA, Maki DG. Effectiveness of preemptive barrier precautions in controlling nosocomial colonization and infection by methicillinresistant Staphylococcus aureus in a burn unit. Am J Infect Control 2006; 34(8):47683.

7. Clinical and Laboratory Standards Institute. Standards Development. Wayne Penssylvania: January2008 [2012-05-10]. Disponible en: http://www.clsi.org

8. Lamikanra A, Olusanya Oi. A longterm study of the nasal carriage of Staphylococcus aureus in the healthy Nigerian students. Trans R Soc Trop Med Hyg 1988 82(3):500-502.

9. Levy Ce, Costa Jc, Lama J, FurlanMls, ToloyRc, PastiMj, et al. Papel epidemiológico das mãos nas infecções hospitalares. Rev. Soc. Bras. Med. Trop 1988 21:89. 
10. Maxwell JG, Ford CR, Peterson DE, Mitchell CR. Longterm study of nasal staphylococci among hospital personal. Am J Surg 1969 118(6):849-854.

11. Callisaya J, Sarmiento Z, Chogue H. Prevalencia de portadores nasales de Staphylococcus aureus en el personal de limpieza del Hospital Obrero. Biofarbo 2007 XV: 55-60

12. Kluytmans J, Van Belkum A, Verbrugh H. Nasal carriage of Staphylococcus aureus: epidemiology, underlying mechanisms, and associated risks. ClinMicrobiol Rev 1997; 10(3): 505-20

13. Montalvo R, Huaroto L, Alvarez J, Ticona E, García Y. Prevalencia de portadores nasales por

Staphylococcusaureusmeticilino resistente en personal de salud del servicio de Cuidados intensivos, Hospital Nacional Dos de Mayo. Rev per epidemiol 2009; 13(2):1-5.

14. Mercado M, Máttar S. Alta resistencia de los microorganismos Nosocomiales en el Hospital San Jerónimo de Montería. Universitas Médica 2003; 44(3):3-6.

15. Londoño J, Ortiz G, Gaviria A. Prevalencia de Staphylococcusaureusresistentea meticilina en personal de la unidad de terapia intensiva de la Clínica Universitaria Bolivariana, Medellín 2004. Infectio 2006;10(3):160-166

16. De Oliveira, B. Estudio longitudinal sobre portador são de Staphylococcus aureus em alunos de um curso de auxiliar de enfermagem. Rev. Soc. Bras. Med. Trop. 1999; 32(4): 395-400.
17. Cárdenas O, Cruz H, Moreno J, Pérez K, Rodríguez D. Determinación de fenotipos y genotipos de resistencia a Macrólidos, Lincosamidas y Estreptograminas B (MLSB) en aislamientos de Staphylococcusaureusresistentes a meticilina asociados a la comunidad (SARM-

AC)entrespaísesLatinoamericanos.

[Revista en Internet] 2009 [citado 201206- 20]; Disponible en: http://www.unbosque. edu.co/files/Archivos/Determinacio n_

Fenotipos_Genotipos_de_Resistencia.p df

18. Cortes JA and Grebo. Implicaciones en Salud Pública de

Staphylococcusaureus Meticilin

o Resistente adquirido en la Comunidad en Bogotá, Colombia. Rev. saludpública [online]. 2007 [citado 2012-06-15]; 9(3):448-454. Disponible $<$ http://www.

scielosp.org/scielo.php?script=sci

arttext \&pid=S012400642007000300013

\&lng=en\&nrm=iso $>. \quad$ ISSN 01240064 .http://dx.doi.org/10.159 o/ S012400642007000300013.

19. Weber T. Community-Associated Methicillin Resistant S. aureus. Clin Infect Dis 2005;41(4):269-72.

20. NaimiTS, LeDell KH, Boxrud DJ, Groom $A V$, Stewars CD, Johnson SK et al. Epidemiology and clonality of community- acquired methicillinr7sistant $S$. aureusin Minnesota, 19961998.Clin Infect Dis 2001; 33(7): 990-6 